9-1984

\title{
Trajectories of an Atomic Electron in a Magnetic Field
}

John B. Delos

William \& Mary, jbdelos@wm.edu

Stephen Knudson

William \& Mary

D. W. Noid

Follow this and additional works at: https://scholarworks.wm.edu/aspubs

Part of the Physics Commons

\section{Recommended Citation}

Delos, John B.; Knudson, Stephen; and Noid, D. W., Trajectories of an Atomic Electron in a Magnetic Field (1984). Physical Review A, 30(3), 1208-1218.

https://doi.org/10.1103/PhysRevA.30.1208

This Article is brought to you for free and open access by the Arts and Sciences at W\&M ScholarWorks. It has been accepted for inclusion in Arts \& Sciences Articles by an authorized administrator of W\&M ScholarWorks. For more information, please contact scholarworks@wm.edu. 


\title{
Trajectories of an atomic electron in a magnetic field
}

\author{
J. B. Delos \\ Physics Department, The College of William and Mary, Williamsburg, Virginia 23185 \\ S. K. Knudson \\ Chemistry Department, The College of William and Mary, Williamsburg, Virginia 23185 \\ D. W. Noid* \\ Theoretical Chemistry Institute, Department of Chemistry, University of Wisconsin, Madison, Wisconsin 53706
}

(Received 12 January 1984)

\begin{abstract}
Classical trajectories of an atomic electron in a magnetic field are calculated for various values of the field strength $B$. Qualitative properties of these trajectories are examined. With use of a scaling law, it is shown that the equations of motion can be written in a form such that they depend upon only one parameter, which may be regarded as a reduced angular momentum (proportional to $L_{z} B^{1 / 3}$ ). For small values of this parameter there is an "elliptical regime" in which the trajectory may be regarded as a Kepler ellipse with orbital parameters that evolve slowly in time. For large values of the parameter there is a "helical regime" in which the electron circles rapidly around a magnetic field line and bounces slowly back and forth along the field. Between these two regimes there is an irregular regime, with "chaotic" orbits and a "transition regime" in which the trajectories can be described in oblate spheroidal coordinates. Bound states persist even at energies above the escape energy, provided that the angular momentum (or field strength) is sufficiently large. With use of action-variable quantization, some formulas for semiclassical energy eigenvalues are given for regimes where the trajectories are regular.
\end{abstract}

\section{INTRODUCTION}

The purpose of this paper is to examine the behavior of an atomic electron in a strong magnetic field. Although there have recently been many experimental and theoretical studies of such systems, ${ }^{1-5}$ much yet remains to be learned. In this paper, we explore the types of classical motion exhibited by an electron under the combined influence of Coulomb and Lorentz forces. Where possible, we give simple approximations that describe the trajectories, and in those cases we give formulas for the semiclassical energy spectrum using the principle of quantization of action. ${ }^{6}$

\section{FUNDAMENTALS}

In a frame of reference that rotates at the Larmor frequency about the direction of the magnetic field, $z$, the Hamiltonian, written in cylindrical coordinates, is

$$
\begin{aligned}
& H=\frac{1}{2 m}\left(p_{\rho}^{2}+p_{z}^{2}\right)-k\left(\rho^{2}+z^{2}\right)^{-1 / 2}+\lambda \rho^{2}+L_{z}^{2} / 2 m \rho^{2}, \\
& k=Z e^{2}, \lambda=e^{2} B^{2} / 8 m c^{2} .
\end{aligned}
$$

The term $-k\left(\rho^{2}+z^{2}\right)^{-1 / 2}$ is the Coulomb potential energy, $L_{z}^{2} / 2 m \rho^{2}$ is the "centrifugal barrier," and $\lambda \rho^{2}$ is the so-called "diamagnetic term," which is proportional to the square of the magnetic field $B$. The Hamiltonian contains four canonical variables $\left(\rho z p_{\rho} p_{z}\right)$ and four parameters $\left(m, k, \lambda, L_{z}\right)$ so it might appear to be impossible to obtain a complete survey of the orbits of the system. In fact, however, it is not difficult: The problem is greatly simplified using a classical scaling law and using Poincaré surfaces of section.

The scaling law was apparently first given by Gajewski ${ }^{7}$ and was used in another way by Robnik. ${ }^{4}$ Let $t$ be the time variable associated with the Hamiltonian (2.1), so that the canonical equations are

$$
d \rho / d t=\partial H / \partial p_{\rho},
$$

etc., and let us define scaled variables $\hat{\rho}, \hat{z}, \hat{p}_{\rho}, \hat{p}_{z}$ as

$$
\begin{aligned}
& \hat{\rho}=\rho / \alpha, \hat{z}=z / \alpha, \\
& \hat{p}_{\rho}=p_{\rho} / \beta, \hat{p}_{z}=p_{z} / \beta, \\
& \hat{t}=t / \gamma, \\
& h \\
& \alpha=(k / 8 \lambda)^{1 / 3}, \\
& \beta=m^{1 / 2} k^{1 / 3}(8 \lambda)^{1 / 6}, \\
& \gamma=(m / 8 \lambda)^{1 / 2} .
\end{aligned}
$$$$
\text { with }
$$

Then the equations of motion in the scaled variables have the form

$$
\begin{aligned}
& d \hat{\rho} / d \hat{t}=\hat{p}_{\rho}, \quad d \hat{z} / d \hat{t}=\hat{p}_{z}, \\
& d \hat{p}_{\rho} / d \hat{t}=-\hat{\rho} /\left(\hat{\rho}^{2}+\hat{z}^{2}\right)^{3 / 2}-\frac{1}{4} \hat{\rho}+\hat{L}^{2} / \hat{\rho}^{3}, \\
& d \hat{p}_{z} / d \hat{t}=-\hat{z} /\left(\hat{\rho}^{2}+\hat{z}^{2}\right)^{3 / 2}
\end{aligned}
$$

These equations of motion contain only one parameter,

$$
\hat{L}=L_{z}(8 \lambda)^{1 / 6} / m^{1 / 2} k^{2 / 3} \text {. }
$$

This means that every trajectory of the Hamiltonian (2.1) 
corresponds to a trajectory of the one-parameter scaled Hamiltonian

$$
\begin{aligned}
\hat{H} & =m H / \beta^{2} \\
& =\left(\hat{p}_{\rho}^{2}+\hat{p}_{z}^{2}\right) / 2-\left(\hat{\rho}^{2}+\hat{z}^{2}\right)^{-1 / 2}+\frac{1}{8} \hat{\rho}^{2}+\hat{L}^{2} / 2 \hat{\rho}^{2}
\end{aligned}
$$

in which the (scaled) mass, electron charge, nuclear charge, and diamagnetic coupling constant $\lambda$ are all equal to unity, and the $z$ component of angular momentum is $\hat{L}$. Hence by surveying the trajectories of (2.7), we learn about all of the trajectories of $(2.1){ }^{8}$

The corresponding semiclassical scaling law has an additional complication. If regular trajectories dominate, allowed semiclassical energy levels can be found by quantizing classical action variables,

$$
J_{i}=\oint_{C_{i}} \sum_{n} p_{n} d q_{n}=M_{i} h
$$

where $C_{i}$ is a suitable closed path, and $M$ is usually an integer or a half-integer. ${ }^{9}$ Equation (2.8) implicitly specifies a relationship between energy and action variables

$$
H=H\left(J_{1}, J_{2} ; m, k, \lambda, L_{z}\right)
$$

(which depends of course on the values of the other parameters contained in the Hamiltonian), and there is a corresponding relationship between allowed energy levels and quantum numbers

$$
E_{M_{1} M_{2}}=H\left(M_{1} h, M_{2} h ; m, k, \lambda, L_{z}\right) \text {. }
$$

Let us define scaled action variables as

$$
\hat{J}_{i}=\oint_{\hat{c}_{i}} \sum_{n} \hat{p}_{n} d \hat{q}_{n}=J_{i} / \alpha \beta
$$

Then the relationship between the scaled Hamiltonian (2.7) and the scaled action variables depends on only one parameter $\hat{L}$ :

$$
\hat{H}=\hat{H}\left(\hat{J}_{1}, \hat{J}_{2} ; \hat{L}\right)
$$

so the allowed values of the scaled energy levels are

$$
\begin{aligned}
\hat{E}_{M_{1}, M_{2}} & =\frac{m}{\beta^{2}} E_{M_{1}, M_{2}} \\
& =\hat{H}\left(M_{1} h / \alpha \beta, M_{2} h / \alpha \beta ; \hat{L}\right) .
\end{aligned}
$$

Hence the parameter $\hat{L}$ determines the overall structure of the spectrum, and the other parameters determine the density of states (see the Appendix).

The second simplification involves use of Poincaré surfaces of section. Examining Eqs. (2.5) for $d \hat{z} / d \hat{t}$ and $d \hat{p}_{z} / d \hat{t}$, one sees that $d^{2} \hat{z} / d \hat{t}^{2}$ is negative if $\hat{z}$ is positive, and positive if $\hat{z}$ is negative. It follows that every trajectory passes through the plane $\hat{z}=0$ at least once for $-\infty<t<\infty$. This means that the full range of trajectories that occur in this system can be found by examining the $\hat{z}=0$ surfaces of section. This is the method we used: For each of a set of values of $\hat{H}$ and $\hat{L}$, twenty random initial conditions were taken for $\hat{\rho}$ and $\hat{p}_{\rho}$, and $\hat{z}$ was set to zero; $\hat{p}_{z}$ was determined using (2.7) for the specified values of $\hat{H}$ and $\hat{L}$, and the equations of motion (2.5) were solved. At each later time that the trajectory again passed through $\hat{z}=0$ with $\hat{p}_{z}>0$, the point $\left(\hat{\rho}, \hat{p}_{\rho}\right)$ was recorded, forming the Poincaré surface of section. By examining about a hundred surfaces of section, we were able to see most of the typical types of behavior that occur in this system.

\section{OVERVIEW OF RESULTS}

In subsequent sections, we shall examine the trajectories in some detail; here we just give a brief overview of their character.

The (scaled) potential energy

$$
\hat{V}(\hat{\rho}, \hat{z})=-\left(\hat{\rho}^{2}+\hat{z}^{2}\right)^{-1 / 2}+\hat{L}^{2} / 2 \hat{\rho}^{2}+\frac{1}{8} \hat{\rho}^{2}
$$

has a minimum at the point $\hat{\rho}_{0}$ which satisfies

$$
\frac{1}{4} \hat{\rho}_{0}^{4}+\hat{\rho}_{0}-\hat{L}^{2}=0
$$

with $\hat{z}_{0}=0$; the value of $\hat{V}$ at that point is

$$
\hat{V}_{0}=-\frac{1}{\hat{\rho}_{0}}+\frac{\hat{L}^{2}}{2 \hat{\rho}_{0}^{2}}+\frac{1}{8} \hat{\rho}_{0}^{2} \equiv \hat{E}_{\min }(\hat{L}) \text {. }
$$

For each $\hat{L}$, this is the minimum possible value of the energy of the system. $\hat{V}(\hat{\rho}, \hat{z})$ also has a "saddle" at $\hat{z}=\infty$, $\hat{\rho}_{s}=(2 \hat{L})^{1 / 2}$, with

$$
\widehat{V}\left(\hat{\rho}_{s}, \infty\right)=\hat{L} / 2 \equiv \hat{E}_{s}(\hat{L}),
$$

$\widehat{E}_{s}$ is the classical escape energy, above which the electron has enough energy to escape from the nucleus (but we shall see that there are some bound trajectories with $\left.\widehat{E}>\hat{E}_{s}\right)$.

If $\hat{E}$ is the energy (i.e., the value of the scaled Hamiltonian) it is convenient to define a dimensionless scaled energy

$$
f=\left[\hat{E}-\hat{E}_{\min }(\hat{L})\right] /\left[\widehat{E}_{s}(\hat{L})-\hat{E}_{\min }(\hat{L})\right] .
$$

At $\widehat{E}=\widehat{E}_{\min }, f$ is zero, and at $\widehat{E}=\widehat{E}_{s}, f$ is unity.

Figure 1 shows the character of typical orbits in various ranges of $f$ and $\hat{L}$, and Fig. 2 shows a collection of Poin-

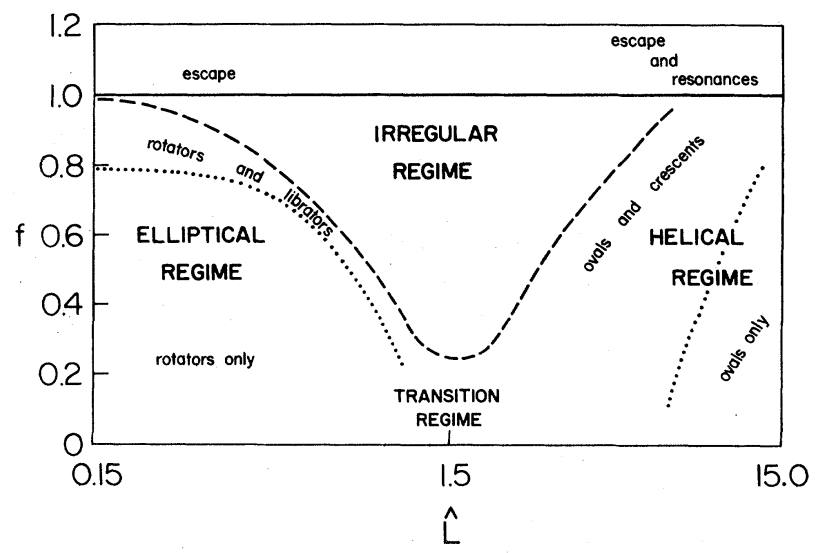

FIG. 1. Types of trajectories that are most common at various values of dimensionless energy $f$, and scaled angular momentum $\hat{L}$. The dimensionless energy is $\left(\hat{E}-\hat{E}_{\min }\right) /\left(\hat{E}_{\text {escape }}-\right.$ $\left.\hat{E}_{\min }\right)$, and the scaled angular momentum is given in Eq. (2.6). One may equivalently regard the horizontal axis as being proportional to the $\frac{1}{3}$ power of the magnetic field. [See also Eq. (8.1).] 

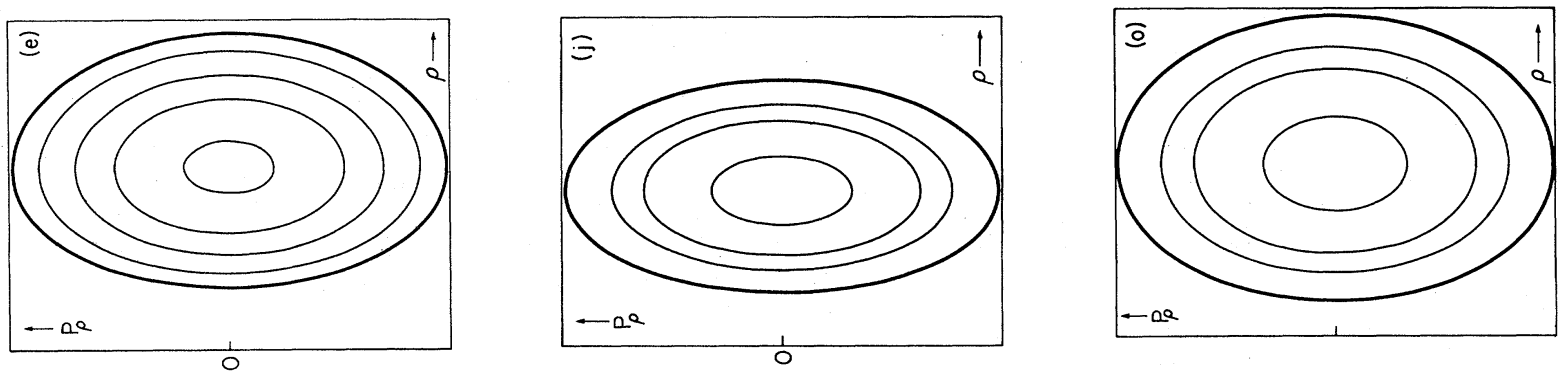

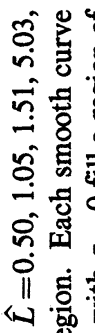

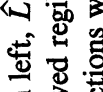

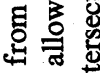
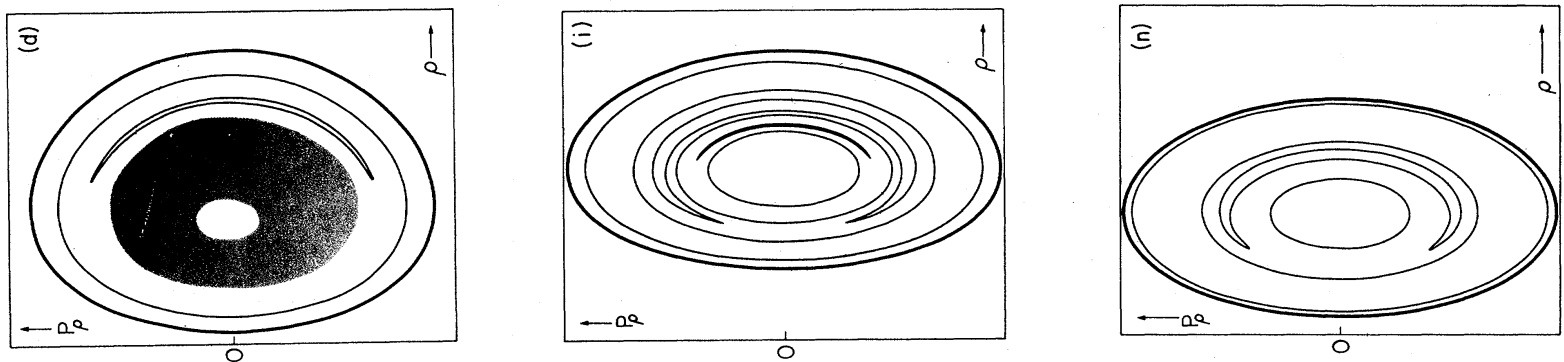

氙

荧

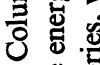

内. 这

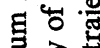

卷

요

홍

00.

도.

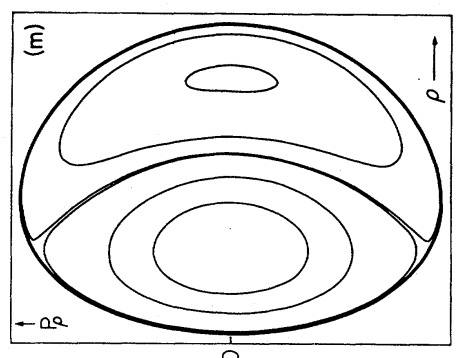

항

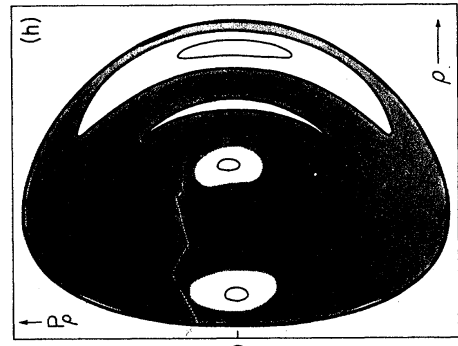

త్ట \&

혏 究

क्षे

ठठ

旁 壱

. $>0$

踏

चै

政

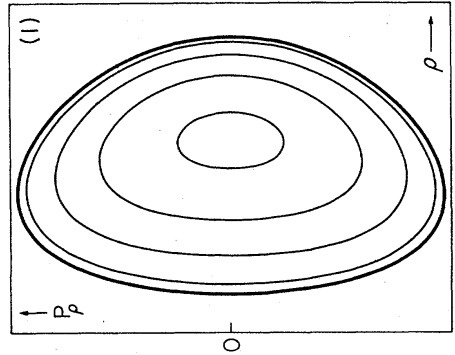

옹.

$>$ 릉

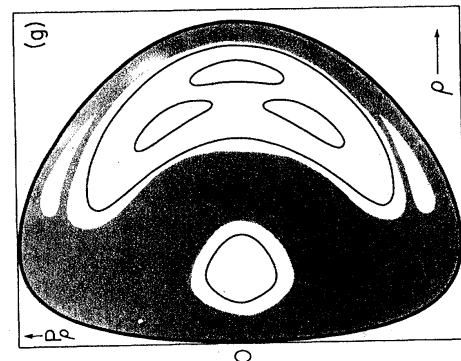

을

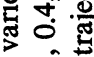

훙혀

ธี

还

눙 홍

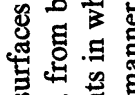

क 仓.

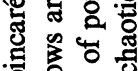

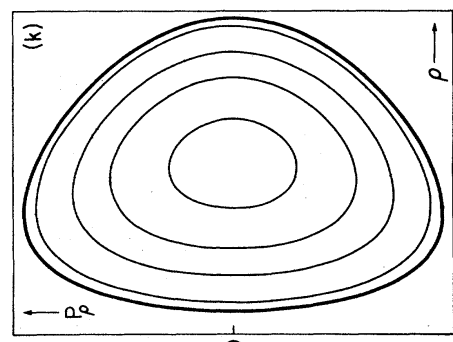

के

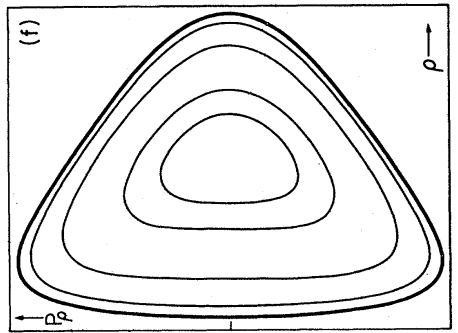

论

훙용

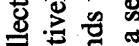

४

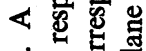

त क 8 है

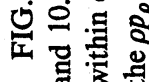


caré surfaces of section. For small $\hat{L}$, which according to the scaling law corresponds to small $L_{z}$, weak magnetic fields, and/or large nuclear charge, trajectories are best described as Kepler ellipses with orbital parameters that slowly vary with time. Many of these trajectories, and their associated quantum states, can be calculated using perturbation theory with the Kepler Hamiltonian as the starting point:

$$
\begin{aligned}
& \hat{H}_{K}^{0}=\frac{1}{2}\left(\hat{p}_{\rho}^{2}+\hat{p}_{z}^{2}\right)-\left(\hat{\rho}^{2}+\hat{z}^{2}\right)^{-1 / 2}+\hat{L}^{2} / 2 \hat{\rho}^{2}, \\
& \hat{H}_{K}^{1}=\frac{1}{8} \hat{\rho}^{2} .
\end{aligned}
$$

Such a description was given, for example, in Ref. 5, and we briefly summarize a few of the results in Sec. IV. We refer to the range of $E$ and $L$ in which the trajectories have an apparent relationship to Kepler ellipses as the elliptical regime. ${ }^{10}$

For large $\hat{L}$, corresponding to large $L_{z}$, to extraordinarily strong magnetic fields (such as might exist on the surface of a neutron star), and/or small (fractional) nuclear charge, the diamagnetic force on the electron exceeds the Coulomb force, so the electron circles around a magnetic field line, and travels slowly back and forth in the $z$ direction. The atom, then, far from being spherical, has the shape of a long cylinder or tube. It has been known for some time that this behavior must exist, but it was not known under what conditions it would occur. We refer to this range of $\hat{E}$ and $\hat{L}$ as the helical regime. In Sec. V below we shall show that the helical trajectories can be described quantitatively using an adiabatic approximation, in which the $\rho$ motion is taken to be rapid and the $z$ motion to be slow.

The helical regime may be said to extend to $f>1$ if $\hat{L}$ is sufficiently large; i.e., even above the escape energy there are bound trajectories with the same helical character. In quantum mechanics these would be quasibound states, or resonances. ${ }^{11(a), 11(b)}$

Between the elliptical and helical regimes, for $f$ not too small, there is an irregular regime, in which the trajectories are in some sense chaotic. This regime extends down to $\hat{L}=0$ in a narrowing strip near $f=1$. It is generally believed that this means that the quantummechanical spectrum of energy levels will also be irregular in at least part of this region. ${ }^{12,13}$

Finally, below the irregular regime, for $f \leqq 0.2$ and $\hat{L} \sim 1.5$, there is an interesting transition regime, in which the trajectories are regular, but they change their character from highly perturbed ellipses to highly perturbed helices. We examine these trajectories in Sec. VII below.

\section{THE ELLIPTICAL REGIME}

Kepler ellipses can be labeled by action variables defined in unscaled variables as follows. $I_{1}$ is the $z$ component of angular momentum $L_{z}, I_{2}$ is the total angular momentum $\left(L_{x}^{2}+L_{y}^{2}+L_{z}^{2}\right)^{1 / 2}$, and $I_{3}$ is the principal action, related to the principal quantum number and equal to $\left(m k^{2} /-2 H_{K}^{0}\right)^{1 / 2}$. The corresponding angle variables represent the longitude of the ascending node $\left(\phi_{1}\right)$, the argument of the perihelion $\left(\phi_{2}\right)$, and the mean anomaly $\left(\phi_{3}\right)$, all of which are described in standard textbooks ${ }^{14}$ (and shown in Fig. 1 of Ref. 5).

In Ref. 5 we showed that there are two types of trajectories in the elliptical regime, that we called "librators" and "rotators." Librating trajectories are ellipses that lie close to either the $+z$ or $-z$ axis, and the argument of the perihelion oscillates in a small range. Rotating trajectories are ellipses that are close to the $x-y$ plane, and the argument of the perihelion decreases monotonically. In Figs. 2(a), 2(f), and 2(k) the concentric loops represent rotators. Detailed descriptions of these trajectories were given earlier.

Rotators are much more prevalent than librators in the elliptical regime: librators can only occur in a small range of the $(f, \hat{L})$ parameter space. We showed in Ref. 5 that librators only exist if the principal action $I_{3}$ exceeds the $z$ component of orbital angular momentum $I_{1} \equiv L_{z}$ by a significant factor:

$$
I_{3} \geq I_{1} \sqrt{5}
$$

which implies

$$
H_{K}^{0} \geq\left(-m k^{2} / 10 L_{z}^{2}\right) \text {. }
$$

Insofar as the (scaled) energy in the elliptical regime is close to the (scaled) Kepler energy,

$$
\widehat{E} \simeq\left(m / \beta^{2}\right) \boldsymbol{H}_{K}^{0},
$$

Eq. (4.2) gives an approximate lower bound on the energy of a librating trajectory: it cannot be much less than

$$
\hat{E} \gtrsim\left[\frac{m}{\beta^{2}}\right]\left[-\frac{m k^{2}}{10 L_{z}^{2}}\right] \gtrsim-\left(10 \hat{L}^{2}\right)^{-1} .
$$

Transforming $\hat{E}$ to $f$ according to Eq. (3.5), this boundary is indicated as the dotted line in Fig. 1; below it there are no librators, while above it librators and rotators coexist (though, even there, there are many more rotators than librators).

It is striking that this boundary parallels the boundary separating the elliptical regime from the irregular regime. In fact, irregular motion first appears near the separatrix between rotators and librators.

\section{THE HELICAL REGIME}

As mentioned earlier, in the helical regime, the electron has an approximately circular motion in $(x, y)$ coordinates and it travels slowly back and forth in the $z$ direction, being confined to finite $z$ by the Coulomb attraction of the nucleus. The majority of the trajectories in this regime can be described quantitatively using an adiabatic approximation that was first given (in quantum mechanics) by Zhilich and Monozon. ${ }^{15-17}$

The approximation is based on the fact that the $z$ motion is slow compared to the $\rho$ motion. Let $S\left(\hat{\rho}, \hat{z}, \widehat{J}_{\rho}, \widehat{J}_{z}\right)$ be the generator of a canonical transformation into action-angle variables. This generator must satisfy the Hamilton-Jacobi equation

$$
\frac{1}{2}\left[\left(\frac{\partial S}{\partial \hat{\rho}}\right]^{2}+\left[\frac{\partial S}{\partial \hat{z}}\right]^{2}\right]+\hat{V}(\hat{\rho}, \hat{z})=\hat{E} .
$$

In the adiabatic approximation we write 


$$
S\left(\hat{\rho}, \hat{z}, \hat{J}_{\rho}, \hat{J}_{z}\right)=S_{1}\left(\hat{\rho}, \hat{J_{\rho}} ; \hat{z}\right)+S_{2}\left(\hat{z}, \hat{J}_{z} ; \hat{J}_{\rho}\right),
$$

where quantities appearing before the semicolons are "variables" and those appearing after the semicolons are "parameters." $S_{1}\left(\hat{\rho}, \hat{J}_{\rho} ; \hat{z}\right)$ is taken to satisfy the equation

$$
\frac{1}{2}\left(\frac{\partial S}{\partial \hat{\rho}}\right)^{2}+V(\hat{\rho} ; \hat{z})=\hat{\epsilon}(\hat{z})
$$

(in which $\hat{z}$ is a parameter). The associated action variable

$$
\begin{aligned}
\hat{J}_{\rho} & =\oint \hat{p}_{\rho} d \hat{\rho} \\
& =\oint\{2[\hat{\epsilon}(\hat{z})-\hat{V}(\hat{\rho} ; \hat{z})]\}^{1 / 2} d \hat{\rho}
\end{aligned}
$$

is taken to be independent of $\hat{z}$; then (5.4) implicitly defines the "energy associated with $\rho$ motion" $\hat{\epsilon}(\hat{z})$. Finally $S_{2}\left(\hat{z}, \hat{J}_{z} ; \hat{J}_{\rho}\right)$ satisfies the equation

$$
\frac{1}{2}\left[\frac{\partial S}{\partial \hat{z}}\right]^{2}+\hat{\epsilon}(\hat{z})=\hat{E},
$$

with which is associated the action variable

$$
\begin{aligned}
\hat{J}_{z} & =\oint \hat{p}_{z} d \hat{z} \\
& =\oint\{2[\hat{E}-\hat{\epsilon}(\hat{z})]\}^{1 / 2} d \hat{z} .
\end{aligned}
$$

Results of this type are perhaps more familiar in quantum mechanics. Semiclassical eigenvalues would be obtained by calculating $\hat{\epsilon}_{n_{p}}(\hat{z})$ using $(5.4)$ and the quantization condition

$$
\hat{J}_{\rho}=\left(\hat{n}_{\rho}+\frac{1}{2}\right) 2 \pi \hbar / \alpha \beta
$$

and then calculating $\hat{E}_{\hat{n}_{\rho}, \hat{n}_{z}}$ using (5.6) and the condition

$$
\widehat{J}_{z}=\left(\hat{n}_{z}+\frac{1}{2}\right) 2 \pi \hbar / \alpha \beta \text {. }
$$

The boundaries of trajectories (i.e., the caustics) are easily calculated in the adiabatic approximation; at each $\hat{z}$, the turning points for the $\hat{\rho}$ motion occur where

$$
\hat{\boldsymbol{\epsilon}}(\hat{z})-\hat{V}(\hat{\rho} ; \hat{z})=0
$$

and the locus of $\hat{z}$ turning points are the lines on which

$$
\widehat{\epsilon}(\hat{z})=\hat{E} \text {. }
$$

In Fig. 3(a) we show one of the numerically computed trajectories with $f=0.8, \hat{L}=34.9$. Surrounding the trajectory are boundaries computed using the adiabatic approximation, as in the preceding paragraph. Clearly the approximation is very accurate for this trajectory. Of course, as $\hat{L}$ decreases, it gets less accurate, as indicated in Fig. 3(b). In the Poincaré surfaces of section, these adiabatic trajectories appear as simple loops [Figs. 2(e), 2(j), and $2(0)$.

Semiclassical energy eigenvalues associated with helical trajectories are easily calculated using the adiabatic approximation, Eqs. (5.7). To show the structure of this spectrum, we have computed it for $B=300 \mathrm{~T}, L=100$, corresponding to $\hat{L}=10.8$ with $\alpha \beta=9.22$. Allowed
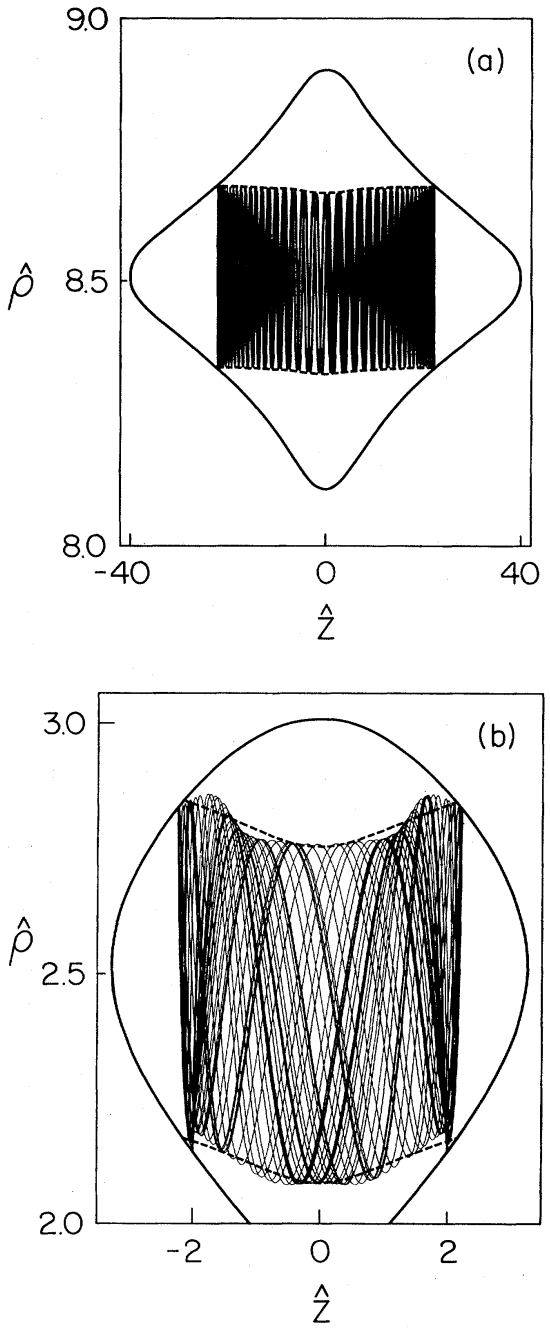

FIG. 3. Two trajectories from the helical regime. (a) Initial conditions for this trajectory were $\hat{\rho}=8.531, \hat{z}=0.0, \hat{p}_{\rho}=0.0$, $\hat{E}=17.4$, with $\hat{L}=34.9$ and $f=0.8$. The trajectory oscillates much more rapidly in $\rho$ than in $z$. The dashed line surrounding the trajectory is the boundary computed using the adiabatic approximation [Eqs. (5.8)]. Evidently the adiabatic approximation is quite accurate for this trajectory. (b) Initial conditions for this trajectory were $\hat{\rho}=2.08, \hat{z}=0.0, \hat{p}_{\rho}=0.0, \hat{E}=1.384$, with $\hat{L}=3.25$ and $f=0.4$. Again the dashed line is the adiabatic approximation for the boundary; the approximation is less accurate in this case.

values of $\hat{\epsilon}_{\hat{n}_{\rho}}(\hat{z})$ are shown as heavy lines in Fig. 4, with the allowed energy levels $\widehat{E}_{\hat{n}_{\rho} \hat{n}_{z}}$ indicated by finer lines. At large $|\hat{z}|$, it is not difficult to show ${ }^{15}$ that $\hat{\epsilon}_{\hat{n}_{\rho}}(\hat{z})$ has the asymptotic form

$\hat{\epsilon}_{\hat{n}_{\rho}}(\hat{z}) \sim\left(\frac{\hat{J}_{\rho}}{2 \pi}+\frac{\hat{L}}{2}\right)-\frac{1}{|\hat{z}|}+\left(\frac{\hat{J}_{\rho}}{2 \pi}+\frac{\hat{L}}{2}\right) \frac{1}{|\hat{z}|^{3}}+\cdots$,

so the $\hat{\boldsymbol{\epsilon}}$ curves become a set of parallel lines with spacing corresponding to the frequency for circular motion in the magnetic field. They have a long-range $|\hat{z}|^{-1}$ attraction, 


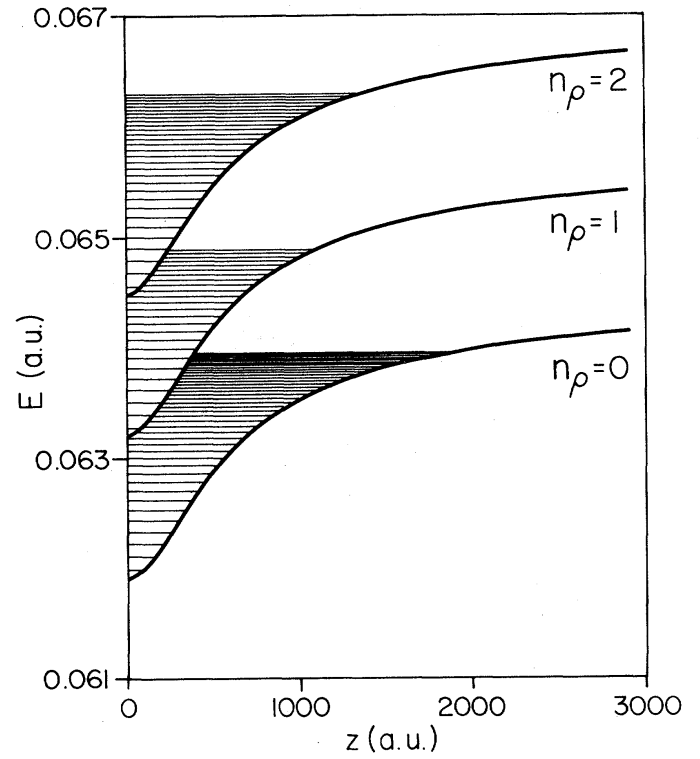

FIG. 4. Spectrum of energy levels in the helical regime, using the adiabatic approximation. Heavy lines are allowed values of $\epsilon_{n}(z)$, which is the energy associated with $\rho$ motion for various values of the $n_{\rho}$ quantum number. Finer lines are allowed energy levels $E_{n_{\rho} n_{z}}$.

and a smooth well near $z=0$. For given $\hat{n}_{\rho}$ the allowed energy levels $E_{\hat{n}_{\rho} \hat{n}_{z}}$ form an oscillatorlike sequence near the bottom of the well, and a Rydberg-type sequence near the top.

For $\hat{n}_{\rho} \neq 0$, many of the states have energies above the quantum-mechanical escape energy, which is the asymptote of the $\hat{\epsilon}_{\hat{n}_{\rho}}=0$ curve. In classical mechanics, such trajectories could be bound, but in quantum mechanics they would be resonances: if nonadiabatic couplings were included, then the electron could escape. As indicated in Fig. 4, the adiabatic approximation predicts that for every $\hat{n}_{\rho} \neq 0$ there is an infinite number of resonant states forming a series that converges to the limit

$$
\widehat{\epsilon}_{\hat{n}_{\rho}}(\hat{z}=\infty)=\left(\hat{n}_{\rho}+\frac{1}{2}\right)+\frac{\hat{L}}{2} .
$$

We confirmed the validity of the adiabatic approximation even for energies above the escape energy by computing exact trajectories in this region and verifying (numerically) that they are bound and quasiperiodic. Certainly there are many resonant states for $\hat{L} \geq 4$. For much smaller $\hat{L}$, we could not find trajectories with $f>1$ for which the electron remained bound. Following the usual arguments about the relationship of quantum states to classical trajectories, this means that if there are any resonance states at small to moderate $\hat{L}$, they would probably have short lifetimes. ${ }^{11(b)}$

In this connection, the discoveries of Garton and Tomkins ${ }^{11(c)}$ are especially interesting. Measuring the photoionization spectrum of atoms in fields of a few $T$, they found a series of broad peaks with spacing equal to $\frac{3}{2}$ the spacing of Landau levels. Edmonds and others ${ }^{11(d)}$ pointed out that there is a set of periodic orbits in which the electron oscillates in the $\rho$ direction with $z=0$, and that at energies corresponding to the ionization threshold, the frequency of these orbits is exactly $\frac{3}{2}$ the Landau frequency. Clearly these orbits are related in some way to the structure of the photoionization spectrum. One might expect that associated with these orbits are quasibound states of the type that occur at larger $\hat{L}$. However, the classical correspondent of a two-dimensional quantum state is not a one-dimensional periodic orbit, but a twodimensional multiply periodic trajectory (unless there is degeneracy, in which case it is a two-dimensional family of periodic orbits). If the quasi-Landau peaks were to correspond to classically bound states, then close to the escape energy there would be trajectories having most of their motion in the $\rho$ direction, but having small stable oscillations about $z=0$. We searched for such trajectories near $f=1.01, \hat{L}=0.03$; none were found. Evidently the quasi-Landau peaks do not correspond to classically bound trajectories of the Hamiltonian (2.1), so in this regard, they are quite different from the resonances that appear at larger $\hat{L}$. In fact, Reinhardt ${ }^{11(d)}$ has interpreted the Garton-Tomkins structure as being related not to resonances, but to a kind of recurrence effect: In his model, ionization of an electron corresponds to production of a wave packet, and the portion of this packet that moves out along $z \simeq 0$ is eventually reflected, so it comes back later to overlap again with the atomic core, and this recurrence produces a peak in the correlation function; the Fourier transform of this correlation function, which is related to the photoabsorbtion cross section, has oscillations similar to those in the observed spectrum.

Finally, there is another type of trajectory that appears in the helical regime when $\hat{L}$ is not too large. These trajectories manifest themselves as crescents on the Poincaré surfaces of section [Figs. 2(d), 2(i), and 2(n)], and, when

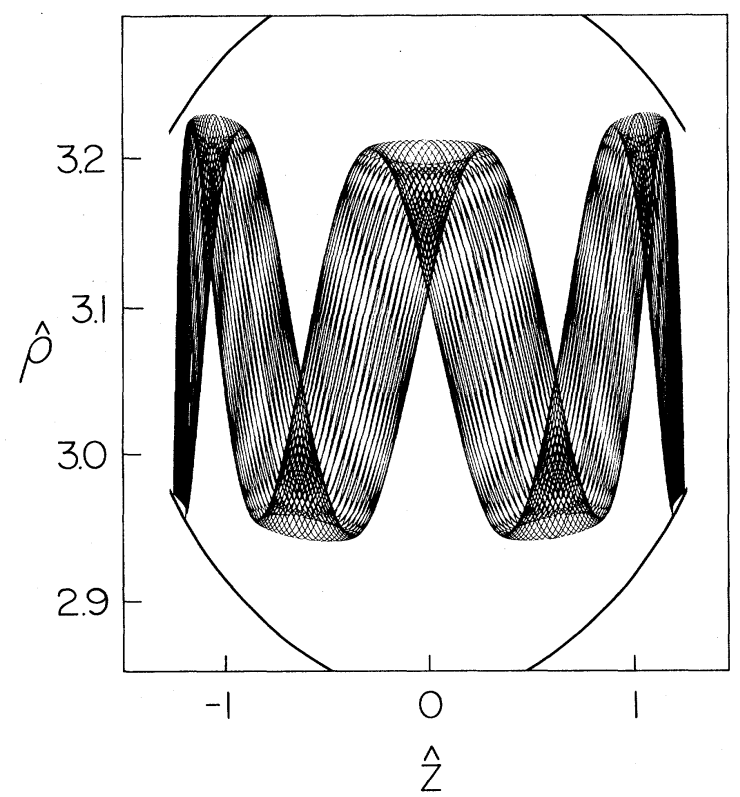

FIG. 5. A trajectory for which the Poincare surface of section has the shape of a crescent. Initial conditions were $\hat{z}=0.0$, $\hat{\rho}=3.212, \hat{p}_{\rho}=0.0, \hat{E}=2.22$, with $\hat{L}=5.035$ and $f=0.1$. 
plotted in $(\rho, z)$ coordinates, they have the shape shown in Fig. 5. They appear to be stable oscillations about a periodic orbit, but we do not yet have any simple description of these trajectories that would tell us when or why they appear. It would be very interesting to find such a description, however, because such trajectories presumably correspond to a distinct class of quantum states, and because irregular motion seems to appear first near the separatrices between various families of regular orbits.

\section{THE IRREGULAR REGIME}

Irregular orbits are identified by the fact that they show no orderly pattern on the Poincaré surface of section. As we move from a regular regime into the irregular regime, disorderly trajectories seem to appear first near separatrices between different types of regular trajectories. They become visible then only in small regions, but as we move further into the irregular regime, they take over most or all of the surface of section until eventually no regular trajectories are visible. Such behavior has also been seen in many other systems. ${ }^{18}$

It follows from these observations that there is no sharp boundary between regular and irregular regimes. The dashed line in Fig. 1 gives a rough indication of the place where regular and irregular trajectories occupy comparable areas in the surface of section. It is interesting that even for $\hat{L}=0.15$, irregular orbits appear and dominate for $f>0.99$, but for $\hat{L} \geq 11$, we have not seen any irregular orbits. ${ }^{19}$

The present studies give the "low-resolution" picture described above. Mathematical and computational studies of other systems have shown the existence of finer structure. $^{18}$ For example, the Kolmogorov-Arnold-Moser theorem asserts that regular trajectories must be dominant under certain conditions, but it permits the possible appearance of irregular trajectories anywhere in a regular regime. On the other hand, even deep in the irregular regime, there may exist small islands of stability containing regular trajectories. Such detailed structure is thought to exist very generally, and one presumes that it exists also in the present case, but our calculations were not carried out to the high level of resolution required to see very fine structure.

Other types of order may also persist in the irregular regime. For example, an irregular trajectory could appear to be very orderly for a long time, especially if the "source" of unstable behavior were highly localized. Figure 6(a) gives an illustration. In this case, the electron could be said to move in an orderly, helical manner until it comes close to the nucleus, where the Coulomb force might be said to "scatter" it onto a different helix. This short-time-scale order is visible on an appropriate $\rho=$ const surface of section [Fig. 6(b)], but not on the $z=0$ surface of section.

Little is known with certainty about the quantum spectrum associated with irregular classical trajectories. Percival $^{12}$ suggested that the spectrum of allowed energy levels would also become "irregular"-no simple pattern to the energy levels would appear, the eigenvalues would be very unstable to small changes in the Hamiltonian, and there would be no selection rules for emission or ab-
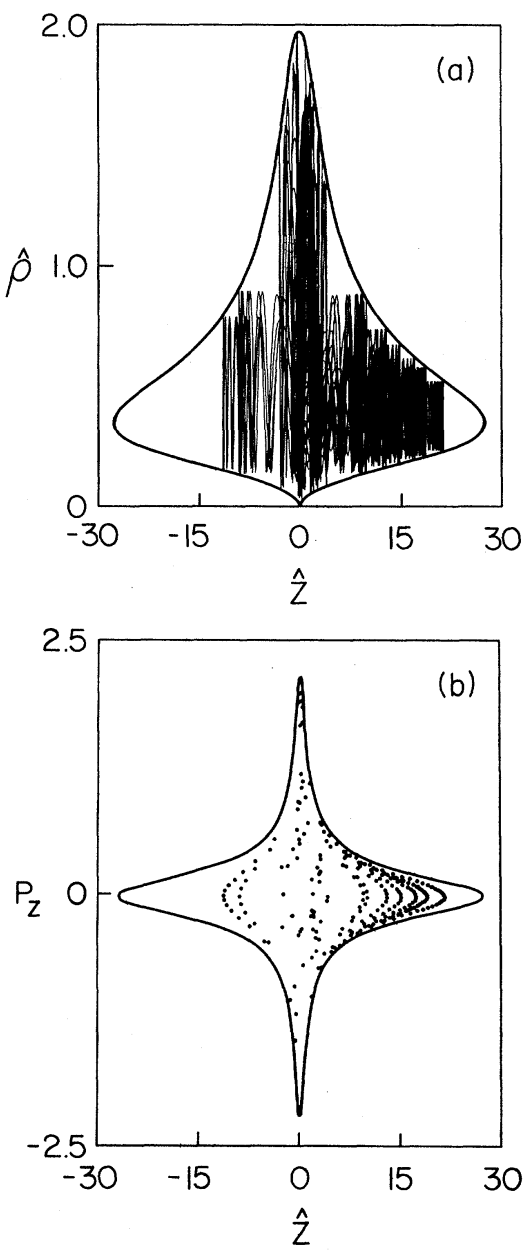

FIG. 6. (a) An irregular trajectory that behaves in an orderly manner over short periods of time. The electron moves approximately on a helix until it comes close to the nucleus. Initial conditions were $\hat{\rho}=0.42, \hat{z}=0.0, \hat{p}_{\rho}=0.0, \hat{E}=-0.432$, and $\hat{L}=0.61$. (b) A surface of section for the single trajectory of Fig. 6(a) at $\hat{\rho}=0.39$. It has the appearance of a succession of helices.

sorbtion of radiation. Berry ${ }^{13}$ refined Percival's suggestions, and he was able to calculate spectra of certain dynamical systems that have ergodic trajectories, and show that they may have irregularities that derive from number theory. However, studies of the Henon-Heiles system have shown that an orderly quantum spectrum may sometimes persist well into a classically irregular regime. $^{20}$

In the present case, one can speculate that part of the spectrum may be irregular, and that orderly patterns based on elliptical or helical pictures of the atom might persist in some part of the irregular regime, but it is better to admit that, except for the density of states, nothing is known about the general structure of the spectrum in this region.

\section{THE TRANSITION REGIME}

Below the irregular regime, for $\hat{L}$ near 1.5 , the trajectories are orderly, but they change their character from elliptical to helical. For sufficiently small $f$, the electron 
stays close to the bottom of the well, and the potential energy can be expanded in a Taylor series, retaining only quadratic terms,

$$
\begin{aligned}
& \hat{V}=\hat{V}_{0}+\frac{1}{2} k_{z} \hat{z}^{2}+\frac{1}{2} k_{\rho}\left(\hat{\rho}-\hat{\rho}_{0}\right)^{2}+\cdots, \\
& k_{z}=\hat{\rho}_{0}^{-3} \\
& k_{\rho}=\frac{1}{4}-\frac{2}{\hat{\rho}_{0}^{3}}+\frac{3 \hat{L}^{2}}{\hat{\rho}_{0}^{4}} .
\end{aligned}
$$

For small $\hat{L}$,

$$
\begin{aligned}
& \hat{\rho}_{0} \simeq \hat{L}^{2}, \\
& k_{\rho} \simeq k_{z} \simeq \hat{L}^{-6},
\end{aligned}
$$

consistent with the degeneracy of the unperturbed Kepler orbits. For large $\hat{L}$,

$$
\begin{aligned}
& \hat{\rho}_{0} \simeq(2 \hat{L})^{1 / 2}, \\
& k_{\rho} \simeq 1, \\
& k_{z} \simeq(2 \hat{L})^{-3 / 2},
\end{aligned}
$$

the frequency of $\rho$ motion approaches the cyclotron frequency $(\omega=e B / m c$ in unscaled variables) while that of $z$ motion becomes small, consistent with the adiabatic picture. For intermediate values of $\hat{L}$, so long as $f$ is sufficiently small, the trajectories are those of a twodimensional harmonic oscillator, separable in $\rho$ and $z$ (Fig. 7).

However, for $f$ even as large as 0.1 , the trajectories have a more complicated and interesting structure. For

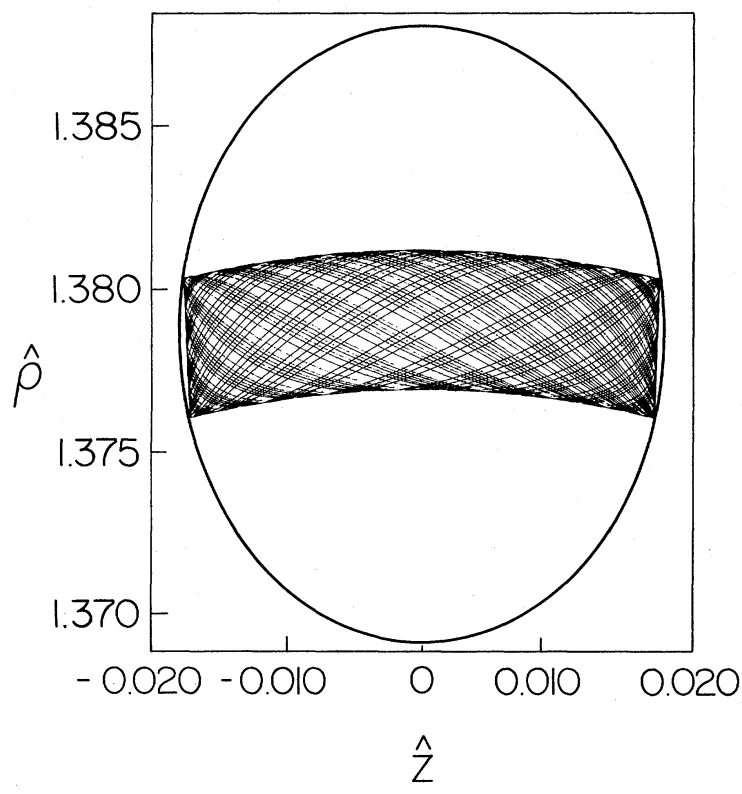

FIG. 7. A trajectory of very low energy in the transition regime. It is like the trajectory of a weakly coupled, twodimensional oscillator, approximately separable in $\rho$ and $z$. Initial conditions were $\hat{\rho}=1.377, \quad \hat{p}_{\rho}=0 ., \quad \hat{z}=0 ., \quad \hat{E}=0.112$, $\hat{L}=1.51$, and $f=0.0001$.
$\hat{L}=1.51, f=0.1$, the Poincare surface of section shows two distinct groups of curves [Fig. 2(m)]. The corresponding trajectories are shown in Fig. 8, and we label them types $E$ and $H$. Type $H$ trajectories disappear when $\hat{L}$ is reduced, and they take over the entire surface of section when $\hat{L}$ is increased. Type $E$ do the opposite. Therefore, we can say that type $H$ are highly perturbed and distorted helical trajectories, while type $E$ are highly distorted elliptical trajectories. However, in both cases, the distortion of the trajectory from the zeroth-order (helical or elliptical) picture is so great that it would be desirable to describe these trajectories in a fundamentally different way.

A useful alternative description of them is obtained with oblate spheroidal coordinates. Let us define

$$
\begin{aligned}
& r_{1}=\left[(\hat{\rho}-d)^{2}+\hat{z}^{2}\right]^{1 / 2}, \\
& r_{2}=\left[(\hat{\rho}+d)^{2}+\hat{z}^{2}\right]^{1 / 2}, \\
& \zeta=\left(r_{2}+r_{1}\right) / 2 d, \\
& \eta=\left(r_{2}-r_{1}\right) / 2 d .
\end{aligned}
$$

For this value of $f$ and $\hat{L}$, the parameter $d$ can be taken to be 1.47 , which is the value of $\hat{\rho}$ at which the separatrix between $H$ and $E$ trajectories crosses the $\hat{p}_{\rho}=0$ axis on the surface of section. The same two trajectories shown in Fig. 8 are replotted in $(\xi, \eta)$ coordinates in Fig. 9. Both of these figures show that there is an approximate separation of variables in these oblate spheroidal coordinates. Such a separation is not apparent from the form of the Hamiltonian. The properties (including semiclassical quantum numbers, energies, and spectra) associated with these two types of trajectories would be expected to be different

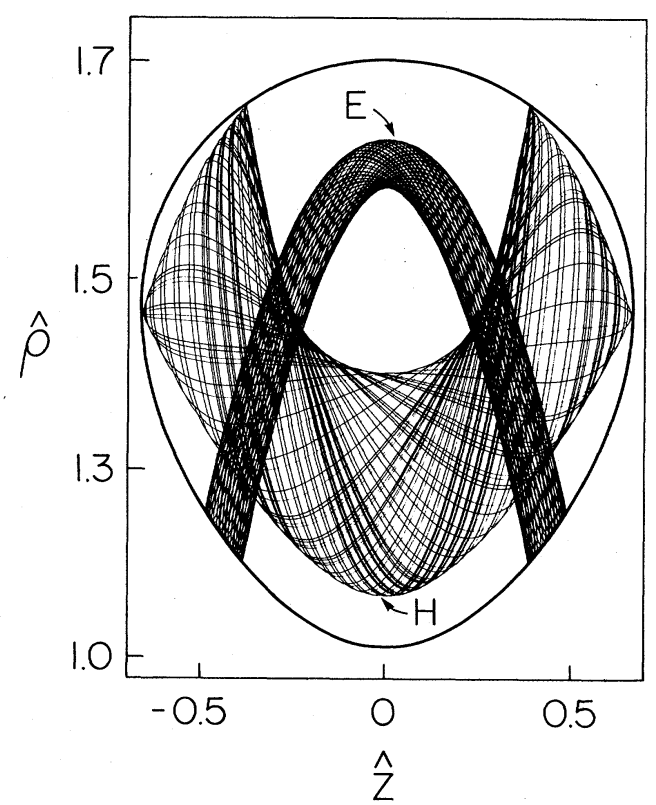

FIG. 8. Two trajectories of low energy in the transition regime. Type $E$ are related to elliptical trajectories, and type $H$ are related to helical ones. Initial conditions were the following: type $E, \hat{\rho}=1.648, \hat{z}=0.0, \hat{p}_{\rho}=0.0, \hat{E}=0.178, \hat{L}=1.51$, $f=0.1$; type $H, \hat{\rho}=1.166$, and other quantities same as above. 


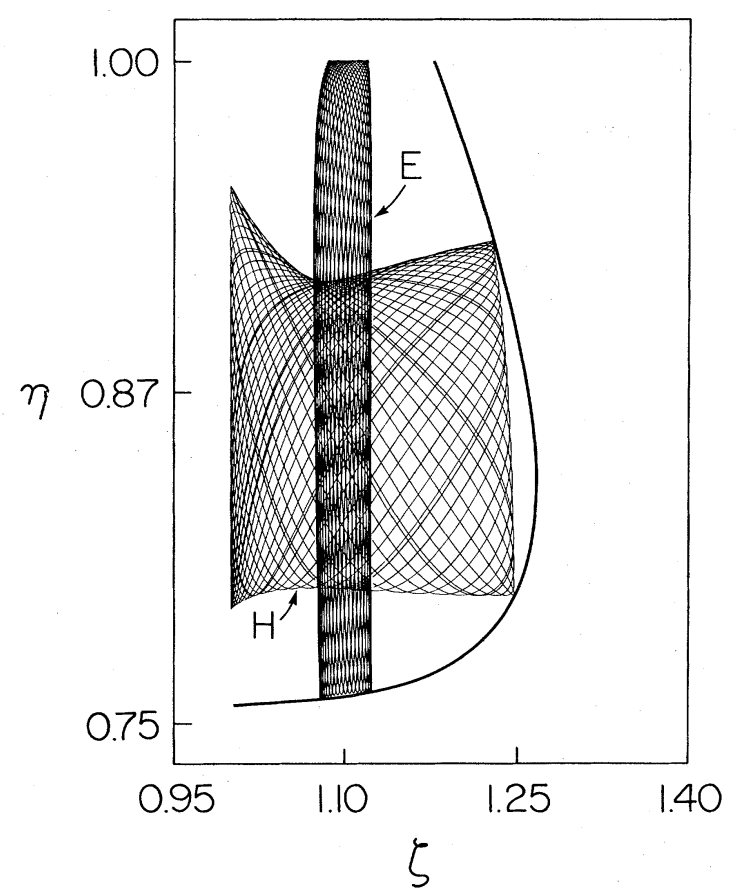

FIG. 9. Trajectories of Fig. 8 replotted in $(\xi, \eta)$ coordinates, Eq. (7.4). If the trajectories formed rectangles, then separation of variables in these coordinates would be appropriate. The figure shows that there is an approximate separation in oblate spheroidal coordinates.

from each other and from those associated with the more familiar helical and elliptical orbits found at high and at low $\hat{L}$. These properties, and their dependence on $\hat{L}$, would be difficult to obtain other than by a trajectory survey.

\section{PROSPECTS}

We have seen that a one-electron atom in a magnetic field shows a wealth of interesting behavior. How much of this might be observed in terrestrial experiments? To answer this, we need to translate our results back into unscaled variables. In atomic units, $e=1, \hbar=1, m=1$, $c=137$, and $B$ is its value in T divided by 1715 . Putting these values into (2.6), we have

$$
L_{z} B^{1 / 3}=61.7 \hat{L},
$$

with $L_{z}$ in units of $\hbar$ and $B$ in T. If an experiment is done with a 10-T field, then

$$
L_{z} \simeq 28 \hat{L} \text {. }
$$

Hence, to see the transition regime and the center of the irregular regime that occur near $\hat{L} \sim 1.5$, we would have to get the electron into states with $L_{z} \simeq 42(\hbar)$.

It is possible to make an atomic beam containing such states, but it would be difficult to perform precise spectroscopic measurements on such a beam. Laser excitation from the ground state typically produces atoms with only one or two units of angular momentum. However, Hulet and Kleppner ${ }^{21}$ recently developed a method for converting a population of $\mathrm{Li}$ atoms with $n=19$ from the $\left|m_{l}\right|=2$ states to $\left|m_{l}\right|=18$ states with essentially
$100 \%$ efficiency. If a beam of such atoms were passed through a magnetic field of $5 \mathrm{~T}$, then the scaled angular momentum $\hat{L}$ would be equal to 0.56 . Examining Figs. 1 and 2, we see that the higher-energy states of such atoms would be well inside the irregular regime, so measurements of the energy spectrum of such atoms might give new information about the relationship between quantum states and irregular classical trajectories.

A totally different approach is easier experimentally, but raises potentially difficult problems of interpretation. If a semiconductor is doped with donor impurities, then the electron associated with that donor feels an effective potential energy $e^{2} / \epsilon r$, where $\epsilon$ is the dielectric constant of the material; also the structure of the energy bands may reduce the effective mass of the electron to a small fraction of its actual mass. A typical value of the effective mass is $m^{*} \sim 0.1 m$, and a typical dielectric constant is $\epsilon \sim 10$. Applying this to the scaling law, we find

$$
\hat{L}=L_{z} B^{1 / 3}\left(\mathrm{\epsilon m} / \mathrm{m}^{*}\right)^{2 / 3} / 61.7 \text {. }
$$

It is then easy to find that the center of the transition regime corresponds to $L_{z} \sim 2.5 \hbar$, so the states of interest are readily observable. In fact, the lowest ten or so have been measured, ${ }^{22}$ and the change of character of the states, from elliptical to helical, seems to be clearly manifested in the energy spectrum. We hope to examine these results more fully in the future.

\section{ACKNOWLEDGMENT}

This research was supported by the National Science Foundation (J.B.D.), the Jeffress Foundation (J.B.D. and S.K.K.), and by the U.S. Department of Energy (D.W.N.) under Contract No. W-7405-eng-26 with Union Carbide Corporation.

\section{APPENDIX: DENSITY OF STATES}

The number of quantum states having energy less than $E$ is related to the corresponding volume in phase space:

$$
\Omega(E)=h^{-2} \int_{H<E} d p_{\rho} d p_{z} d \rho d z
$$

and the density of states is

$$
N(E)=d \Omega / d E \text {. }
$$

If we define a volume in the scaled phase space as

$$
\widehat{\Omega}(\widehat{E})=\int_{\hat{H}<\hat{E}} d \hat{p}_{\rho} d \hat{p}_{z} d \hat{\rho} d \hat{z}
$$

then

$$
\begin{aligned}
N(E) & =(\alpha \beta / h)^{2}(d \hat{E} / d E) \hat{N}(\hat{E}) \\
& =\left(m \alpha^{2} / h^{2}\right) \hat{N}(\widehat{E})
\end{aligned}
$$

where

$$
\hat{N}(\hat{E})=d \widehat{\Omega} / d \hat{E} .
$$

The scaled volume $\widehat{\Omega}(\widehat{E})$ is easily calculated by integrating first over momenta, then over $\widehat{z}$, and last over $\hat{\rho}$, 


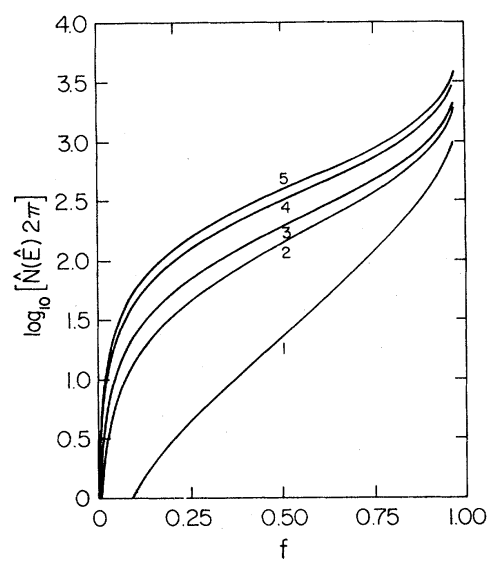

FIG. 10. Density of states. Shown is $\log [2 \pi \hat{N}(\hat{E})]$ as a function of reduced energy $f$. Curves $1-5$ are, respectively, $\hat{L}=0.46,1.05,1.51,5.04,10.8$.

$$
\widehat{\Omega}(\hat{E})=\int_{\hat{\rho}_{1}}^{\hat{\rho}_{2}} d \hat{\rho} \int_{\hat{z}_{1}}^{\hat{z}_{2}} d \hat{z} \iint d \hat{p}_{\rho} d \hat{p}_{z} .
$$

The integral over momenta includes all values such that

$$
\frac{1}{2}\left(\hat{p}_{\rho}^{2}+\hat{p}_{z}^{2}\right) \leq \hat{E}-\hat{V}(\hat{\rho}, \hat{z})
$$

which is a circle of area $\pi 2[\hat{E}-\hat{V}(\hat{\rho}, \hat{z})]$, so

$$
\widehat{\Omega}(\hat{E})=\int_{\hat{\rho}_{1}}^{\hat{\rho}_{2}} d \hat{\rho} \int_{\hat{z}_{1}}^{\hat{z}_{2}} d \hat{z} 2 \pi[\hat{E}-\hat{V}(\hat{\rho}, \hat{z})] .
$$

The integral over $z$ has endpoints $\widehat{z}_{1}<0, \hat{z}_{2}>0$ such that $\widehat{V}\left(\hat{\rho}, \hat{z}_{1}\right)=\widehat{E}$; i.e.,

$$
\hat{z_{i}}= \pm\left\{\left[\hat{E}-\left[\frac{\hat{L}^{2}}{2 \hat{\rho}^{2}}+\frac{1}{8} \hat{\rho}^{2}\right]\right]^{-2}-\hat{\rho}^{2}\right\}^{1 / 2}
$$

Defining

$$
\hat{V}_{m}(\hat{\rho})=\frac{\hat{L}^{2}}{2 \hat{\rho}^{2}}+\frac{1}{8} \hat{\rho}^{2},
$$

one quickly finds that

$$
\begin{aligned}
\widehat{\Omega}(\hat{E})=2 \pi \int_{\hat{\rho}_{1}}^{\hat{\rho}_{2}}[ & {\left[\hat{z_{2}}(\hat{\rho})-\hat{z}_{1}(\hat{\rho})\right]\left[\hat{E}-\hat{V}_{m}(\hat{\rho})\right] } \\
& \left.+\ln \left(\frac{1+\hat{z}_{2} /\left(\hat{z}_{2}^{2}+\hat{\rho}^{2}\right)^{1 / 2}}{1+\hat{z}_{1} /\left(\hat{z}_{1}^{2}+\hat{\rho}^{2}\right)^{1 / 2}}\right) d \rho\right] .
\end{aligned}
$$

This integral was evaluated and differentiated numerically to obtain $\widehat{N}(\hat{E})$. In Fig. 10 is shown for various values of $\hat{L}$ the scaled density of states $\hat{N}(\hat{E})$ plotted as a function of dimensionless energy, $f$. We note that as $f \rightarrow 1$, the density of states always goes to infinity, because of the long-range Coulomb interaction.
*Permanent address: Chemistry Division, Oak Ridge National Laboratory, Oak Ridge, TN 37830 and Department of Chemistry, University of Tennessee, Knoxville, TN 37916.

${ }^{1}$ L. I. Schiff and H. Snyder, Phys. Rev. 55, 59 (1939); J. M. Wadehra, Astrophys. J 226, 372 (1978); V. Canuto and D. C. Kelly, Astrophys. Space Sci. 17, 277 (1972); J. J. Labarthe, J. Phys. B 14, 1467 (1981); C. W. Clark, Phys. Rev. A 24, 605 (1981); A. R. P. Rau, ibid. 16, 613 (1977); H. Hasegawa and R. E. Howard, J. Phys. Chem. Solids 21, 179 (1961); R. J. E1liott and R. Loudon, ibid. 15, 196 (1960).

${ }^{2}$ M. L. Zimmerman, J. C. Castro, and D. Kleppner, Phys. Rev. Lett. 40, 1083 (1978); J. C. Castro, M. L. Zimmerman, R. G. Hulet, D. Kleppner, and R. R. Freeman, ibid. 45, 1780 (1980); D. Kleppner, M. G. Littman, and M. L. Zimmerman, Sci. Am. 244, no. 5, 130 (1981).

${ }^{3}$ D. R. Herrick, Phys. Rev. A 26, 323 (1982); R. Cohen, J. Lodenquai, and M. Ruderman, Phys. Rev. Lett. 25, 467 (1970); M. Ruderman, ibid. 27, 1306 (1971); E. G. Flowers, J.-F. Lee, M. A. Ruderman, P. G. Sutherland, W. Hillebrandt, and E. Müller, Astrophys. J. 215, 291 (1977); R. H. Garstang, Rep. Prog. Phys. 40, 105 (1977); J. C. Gay, NATO Advanced Study Institute on Photophysics and Photochemistry in the Vacuum Ultraviolet report (unpublished); E. A. Solov' ev, Zh. Eksp. Teor. Fiz. Pis'ma Red 34, 278 (1981) [Sov. Phys.-JETP Lett. 34, 237 (1981)]; H. Friedrich, Phys. Rev. A 26, 1827 (1982); S. H. Patil, J. Phys. B 15, 1161 (1982); J. A. C. Gallas, E. Gerck, and R. F. O’Connell, Phys. Rev. Lett. 50, 324 (1983).
${ }^{4} \mathrm{~A}$ study somewhat similar to this one was undertaken by $\mathbf{M}$. Robnik, J. Phys. A 14, 3195 (1981); he was primarily interested in showing the existence of regular and irregular classical motion. Our primary interest is in the properties of the regular trajectories, so we examine them in much more detail than he did.

5J. B. Delos, S. K. Knudson, and D. W. Noid, Phys. Rev. Lett. 50, 579 (1983); Phys. Rev. A 28, 7 (1983); S. K. Knudson and D. W. Noid, Chem. Phys. (to be published).

${ }^{6}$ A. Einstein, Verh. Dtsch. Phys. Ges. 19, 82 (1917); J. B. Keller, Ann. Phys. (N.Y.) 4, 180 (1958); R. A. Marcus, Discuss. Faraday Soc. 55, 34 (1973); D. W. Noid and R. A. Marcus, J. Chem. Phys. 62, 2119 (1975); V. P. Maslov and M. V. Fedoriuk, Semiclassical Approximation in Quantum Mechanics (Reidel, Boston, 1981); D. W. Noid, M. L. Koszykowski, and R. A. Marcus, Annu. Rev. Phys. Chem. 32, 267 (1981).

${ }^{7}$ R. Gajewski, Physica (Utrecht) 47, 575 (1970).

${ }^{8}$ For $m=1$ and $k=1$ in atomic units, with $L_{z}$ measured in units of $\hbar$ and $B$ in T, $\hat{L}=L_{z} B^{1 / 3} / 61.7$.

${ }^{9}$ Such quantization conditions have been proved to be applicable for separable or integrable systems [see, for example, V. P. Maslov and M. V. Fedoriuk (Ref. 6)]. Experience has shown that they are also appropriate for nonintegrable systems, at least in those regions in which regular trajectories are strongly dominant.

${ }^{10}$ In Ref. 5 we identified the "linear regime" as that range of $E$ and $L$ in which $H_{K}^{1}$ is negligible, and only Larmor effects, linear in $B$, are significant. Likewise, the "quadratic regime" 
was said to be that region in which first-order perturbation theory based on (3.6) is adequate; i.e., it is that region in which energy levels are approximately proportional to $B^{2}$. The linear and quadratic regions are thus contained within the elliptical regime.

11(a) D. W. Noid, S. K. Knudson, and J. B. Delos, Chem. Phys. Lett. 100, 367 (1983); (b) M.-C. Chu and H. Friedrich, Phys. Rev. A 29, 675 (1984); (c) W. R. S. Garton and F. S. Tomkins, Astrophys. J. 158, 839 (1969); (d) A. R. Edmonds, J. Phys. (Paris) Colloq. 31, C4 (1970); A. R. P. Rau, Comments At. Mol. Phys. 10, 19 (1980); A. F. Starace, J. Phys. B 6, 585 (1973); W. P. Reinhardt, ibid. 16, L635 (1983).

12I. C. Percival, J. Phys. B 6, L229 (1973); Adv. Chem. Phys. 36, 1 (1977).

${ }^{13}$ M. V. Berry, Philos. Trans. R. Soc. London Ser. A 287, 237 (1977); J. Phys. A 10, 2083 (1977).

${ }^{14}$ For example, H. Goldstein, Classical Mechanics, 2nd ed. (Addison-Wesley, Reading, Mass., 1980).

${ }^{15}$ A. G. Zhilich and B. S. Monozon, Fiz. Tverd. Tela (Leningrad) 8, 3559 (1966) [Sov. Phys.-Solid State 8, 2846 (1967)].

${ }^{16} \mathrm{~A}$ different approximation was used by $\mathrm{G}$. Wunner and $\mathbf{H}$. Ruder, Astrophys. J. 242, 828 (1980). Although they called their method an "adiabatic" approximation, we would not use that terminology. They assumed that the wave function is separable in $\rho$ and $z$ coordinates, $\psi=u_{1}(\rho) u_{2}(z)$. Our adiabatic approximation corresponds to $\psi=\phi_{1}(\rho ; z) \phi_{2}(z)$.

${ }^{17}$ Different types of adiabatic approximation were suggested by A. F. Starace and G. L. Webster, Phys. Rev. A 19, 1629 (1979); since the approximations are not based on the idea that the $\rho$ motion is fast and the $z$ motion is slow, they do not appear to be suitable for helical trajectories.

${ }^{18}$ M. Henon and C. Heiles, Astron. J. 69, 73 (1964); G. H. Walker and J. Ford, Phys. Rev. 188, 416 (1969).

${ }^{19}$ A. Harada and H. Hasegawa, J. Phys. A 16, L259 (1983) report irregular orbits also for $\hat{L}=0$.

${ }^{20}$ R. T. Swimm and J. B. Delos, J. Chem. Phys. 71, 1706 (1979); C. Jaffe and W. P. Reinhardt, ibid. 77, 5191 (1983); R. B. Shirts and W. P. Reinhardt, ibid. 77, 5204 (1983); D. W. Noid, M. L. Koszykowski, M. Tabor, and R. A. Marcus, ibid. 72, 6169 (1980).

${ }^{21}$ R. G. Hulet and D. Kleppner, Phys. Rev. Lett. 51, 1430 (1983).

${ }^{22}$ N. Kuroda, Y. Nishina, H. Hori, and M. Date, Phys. Rev. Lett. 48, 1215 (1982); A. K. Ramdas and S. Rodriquez, Rep. Prog. Phys. 44, 1297 (1981). 

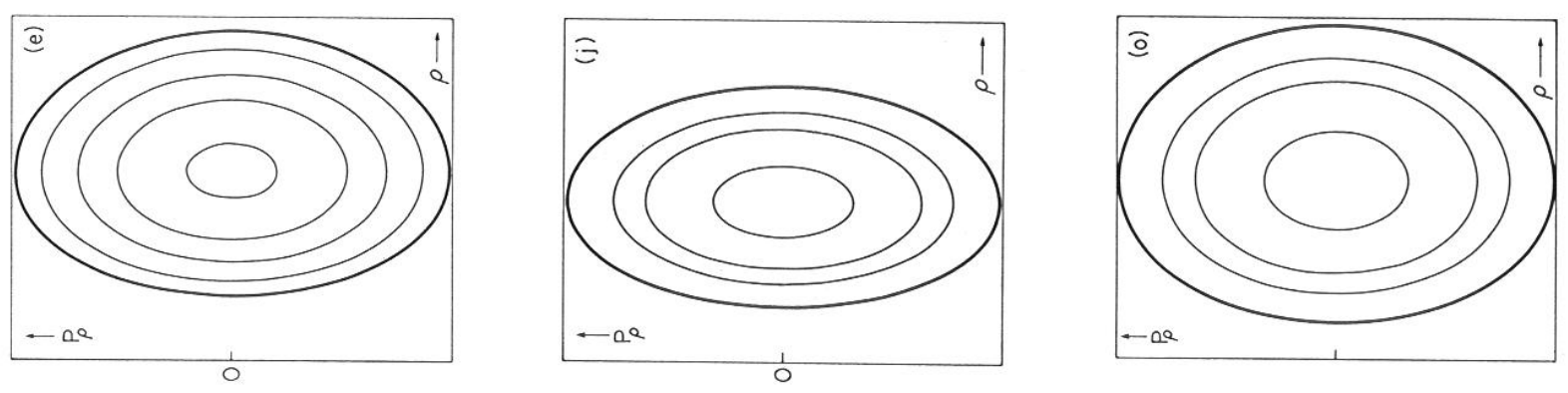

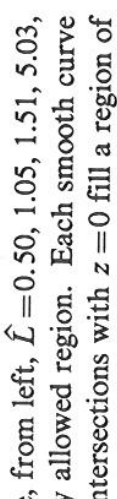
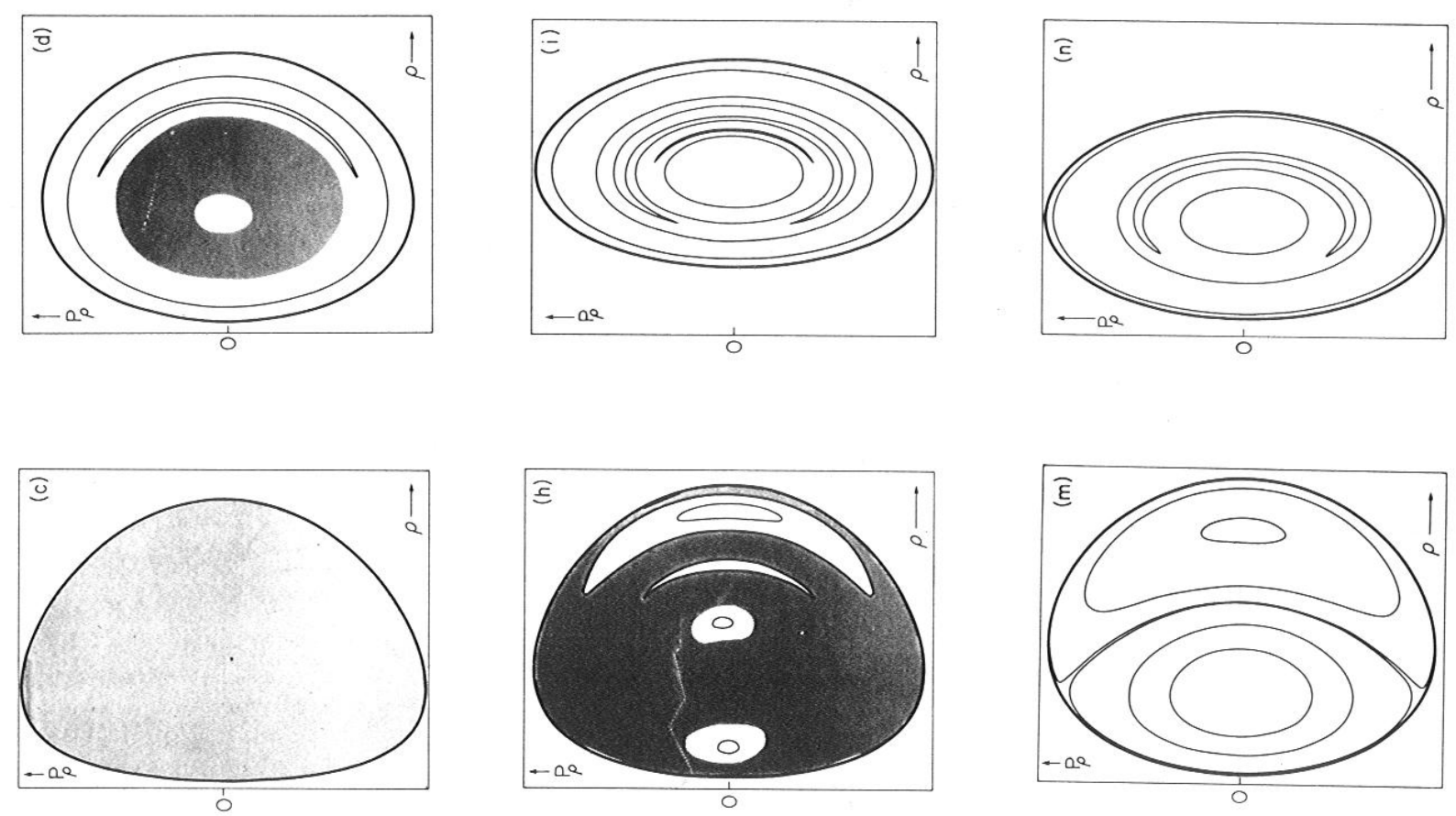

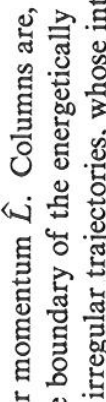

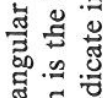

ำ 으.

]ु

वृ

$4 . \Xi 0$

के

वृ

总吉壱

至

텅

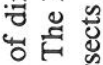

\& $\infty$ 岌

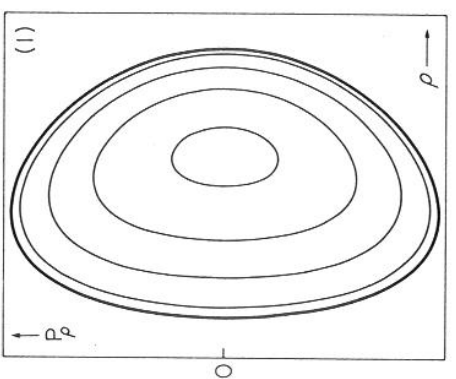

$>$

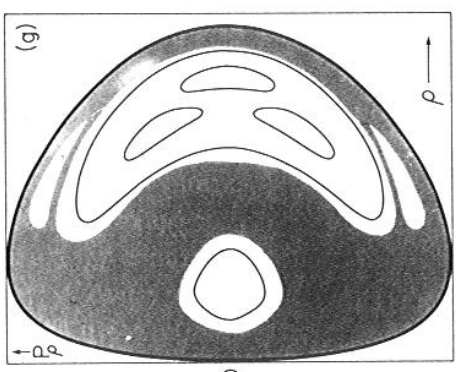

路

芯芯

둔

.ํํㅇ

\&

范

兽要

क स

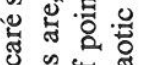
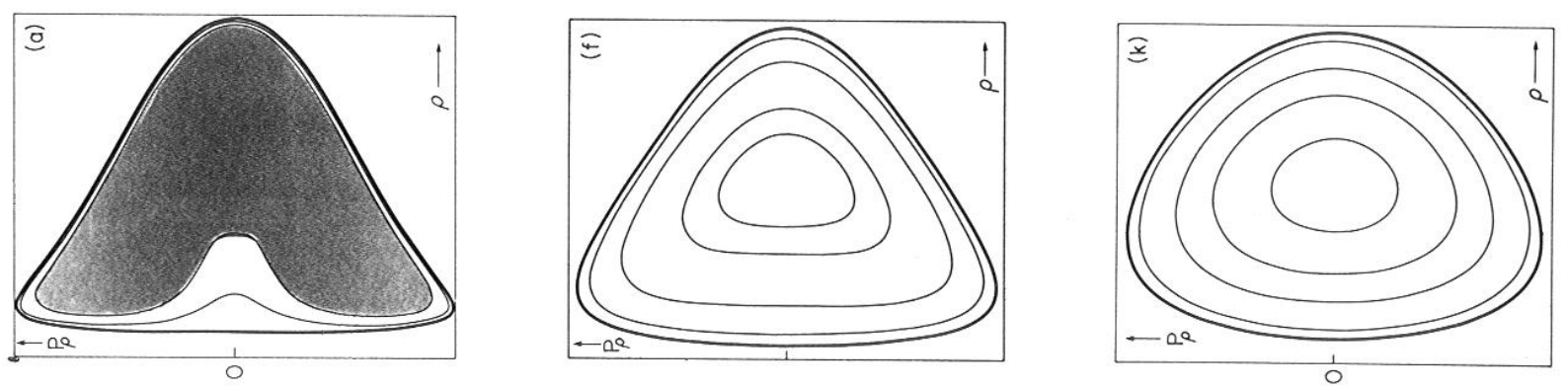

.

녕에

동용

ठै.

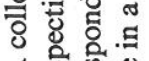

४ क्षे

ن

نั 